\title{
Informe de endoscopia ginecológica
}

\author{
Ramiro Argüello Argüello*; Jaime Ferro Camargo**; Pío Iván Gómez Sánchez***; Sergio Augusto Parra Duarte*****
}

RESUMEN: El desarrollo de la Endoscopia ginecológica en los últimos años ha hecho que los procedimientos operatorios laparohisteroscópicos sean cada vez más frecuentes. Los informes escritos utilizados generalmente se orientan hacia la descripción diagnóstica y carecen de precisión en la descripción de la parte operatoria y en el resultado finaI. Como el pronóstico y el tratamiento de una determinada paciente frecuentemente pueden basarse únicamente en lo anotado en el informe, es indispensable utilizar un documento descriptivo que sea lo más exacto posible. Se presenta un modelo de Informe en Endoscopia GinecoIógica, recomendado por la Comisión permanente en Endoscopia Ginecológica de la Sociedad Colombiana de Obstetricia y Ginecología y se explican sus ventajas.

PALABRAS CLAVES: Laparoscopia, histeroscopia, endoscopia ginecológica, informe, documentación.

SUMMARY: The development of Endoscopic Surgery in gynecology in recent years has made the laparo-histeroscopic surgery much more frequent every day. The documentation forms generally used are aimed toward diagnostic description and have not enough accuracy in the surgical descriptions or in the final results. Since the prognostic and the treatment of a patient could be based only in the documentation form, is indispensable to use a form the most exact as possible A documentation form

for Endoscopic Gynecology recommended by the Comisión permanente en Endoscopia Ginecológica of the Sociedad Colombiana de Obstetricia y Ginecología is presented and its advantages explained.

KEY WORDS: Laparoscopy, histeroscopy, gynecological endoscopy, documentation, documentation form.

\section{Introducción}

Desde 1987, cuando Phillippe Mauriat realizó la primera Colecistectomía por Laparoscopia en Lyon, Francia (1), los procedimientos quirúrgicos realizados mediante técnicas que podemos llamar de videoendoscopia se han venido multiplicando de manera asombrosa en

\footnotetext{
* Coordinador de la Comisión Permanente en Endoscopia Ginecológica de la Sociedad Colombiana de Obstetricia y Ginecología. Certificación de Experto en Endoscopia Ginecológica dado por la Sociedad Colombiana de Obstetricia y Ginecología. Director Científico de la Unidad de Endoscopia Ginecológica y Dolor Pélvico. UNEGIN Ltda.

Presidente de la Sociedad Colombiana de Obstetricia y Ginecología. Miembro Comité Científico de la Federación Internacional de Sociedades de Obstetricia y Ginecología -FIGO-. Miembro de la Comisión Permanente en Endoscopia Ginecológica de la Sociedad Colombiana de Obstetricia y Ginecología. Certificación de Experto en Endoscopia Ginecológica dado por la Sociedad Colombiana de Obstetricia y Ginecología.

Profesor Asociado y Director del Departamento de Obstetricia y Ginecología de la Universidad Nacional de Colombia. Secretario de la Asociación Bogotana de Obstetricia y Ginecología. Miembro de la Comisión Permanente en Endoscopia Ginecológica de la Sociedad Colombiana de Obstetricia y Ginecología. Certificación de Experto en Endoscopia Ginecológica dado por la Sociedad Colombiana de Obstetricia y Ginecología.

**** Miembro de la Comisión Permanente en Endoscopia Ginecológica de la Sociedad Colombiana de Obstetricia y Ginecología. Certificación de Experto en Endoscopia Ginecológica dado por la Sociedad Colombiana de Obstetricia y Ginecología. Coordinador Académico de Pregrado y Postgrado del Hospital San José.
}

todas las especialidades (2). La Ginecología no ha sido la excepción, de tal forma que en Colombia ya se cuentan 26 procedimientos ginecológicos operatorios laparoscópicos y 5 histeroscópicos operatorios, incluidos en el Manual de Tarifas y Procedimientos del Instituto de Seguros Sociales de 1996 (3).

La mayoría de los Informes de Endoscopia Ginecológica utilizados en Colombia presentan un formato similar, diseñado en épocas anteriores al desarrollo de la cirugía por Laparoscopia, generalmente orientados para usar en laparoscopia diagnóstica y esterilización tubárica por laparoscopia, sin utilización de técnicas de video y por consiguiente sin posibilidades de realizar procedimientos operatorios complejos. Este tipo de formato presenta limitaciones importantes en el momento de anotar adecuadamente cualquier procedimiento operatorio realizado. Además es muy frecuente que no incluyen datos sobre Histeroscopia diagnóstica u operatoria.

En 1993 la Unidad de Endoscopia Ginecológica y Dolor Pélvico, UNEGIN, diseñó un Informe de Videolaparoscopia y Videohisteroscopia que contemplaba la posibilidad de anotar los procedimientos operatorios, además de los histeroscópicos realizados. Este Informe lo han venido utilizando desde entonces en más de 700 laparoscopias y se adoptó para su uso, con autorización, en el Instituto Materno Infantil. En Santafé de Bogotá este modelo de informe se está difundiendo lentamente e informes con características muy similares se encuentran en uso en otras instituciones con Unidades de Endoscopia Ginecológica. 
A pesar de representar un avance en cuanto a la facilidad de informar de una manera adecuada y objetiva los procedimientos operatorios efectuados, hemos detectado las siguientes fallas en el mismo, las cuales pueden aplicarse a la mayoría de informes utilizados en la actualidad por las diferentes unidades de Endoscopia Ginecológica en nuestro medio:

1. No se informan antecedentes de importancia que pudieran influir en el tipo de punción inicial que se le debe practicar a la paciente (por ejemplo técnica abierta si hay antecedentes de peritonitis).

2. Aunque es posible dibujar adecuadamente los hallazgos patológicos encontrados inicialmente, es muy difícil dibujar los procedimientos operatorios practicados y, más importante, el resultado postoperatorio final.

3. Se utiliza mucho tiempo anotando y describiendo hallazgos normales.

4. No es fácil hacer una correlación adecuada entre el diagnóstico y el procedimiento operatorio de una patología específica (ejemplo. Hidrosálpinx derecho en el cual se realizó una salpingoneostomía).

5. No hay espacio para anotar los controles postoperatorios con las posibles complicaciones, si las hubiere, y los resultados de patología.

Analizando la información anterior la Comisión permanente en Endoscopia Ginecológica de la Sociedad Colombiana de Obstetricia y Ginecología diseñó un nuevo modelo de Informe de Endoscopia Ginecológica, tomando como base (con autorización para su modificación), un formato desarrollado recientemente por la Unidad de Endoscopia Ginecológica y Dolor Pélvico, UNEGIN.

Como puntos importantes para destacar en este modelo están los siguientes:

1. Se anota si se filmó el procedimiento en cinta de video.

2. Se pueden anotar antecedentes de importancia que influyan en la técnica o en aspectos específicos de la Endoscopia Ginecológica. Esto es importante para los sitios en los cuales se maneja un volumen grande de pacientes, o en sitios en donde el médico que valora inicialmente a las pacientes puede no ser el mismo que realiza la cirugía, como por ejemplo en los hospitales universitarios. En estos casos es conveniente recordarle al cirujano este punto, de manera rápida, inmediatamente antes de la cirugía.

3. Se añade un tercer gráfico en el cual es posible dibujar los procedimientos operatorios y se da un código visual para la Fulguración, el Corte y la Sutura. Este dibujo permite apreciar en conjunto los procedimientos realizados y los resultados finales obtenidos.

4. Se agiliza la parte escrita del informe dando la posibilidad de anotar únicamente los hallazgos anormales.

Por ejemplo: si en la trompa izquierda lo único encontrado como anormal es una alteración en la fimbria, se colocará $\mathbf{V}$ en todos los demás recuadros y $\mathbf{X}$ en el correspondiente al Número 3, Fimbria; en el espacio para describir lo anormal de la trompa izquierda basta anotar 3: aglutinación parcial, etc.

Si por ejemplo no se valoró la permeabilidad se dejará en blanco el recuadro correspondiente.
Otras de las ventajas de este modeIo, además del ahorro de tiempo al describir sólo lo anormal, es que acostumbra al cirujano a evaluar las principales características de cada región.

5. En la sección de Diagnósticos permite hacer una correlación directa e inmediata con el procedimiento operatorio realizado para cada patología específica. Por ejemplo si se encontraron adherencias ováricas derechas se puede anotar a continuación: ovariolisis derecha total o parcial.

6. Se coloca el recuadro para Descripción Operatoria inmediatamente a continuación de la descripción del diagnóstico. En este espacio se debe anotar: número, tamaño y localización de punciones, procedimientos realizados, fuente de energía utilizada, complicaciones, toma de muestras para estudio anatomopatológico.

7. Se coloca un recuadro para Histeroscopia diagnóstica y/o operatoria.

8. Se añade un recuadro en donde es posible anotar varios controles postoperatorios, complicaciones tardías encontradas y resultado anatomopatológico.

\section{Discusión}

El avance de las técnicas Endoscópicas Ginecológicas en los últimos años ha hecho que cada vez sea mayor el número de procedimientos que se realizan mediante videolaparoscopia (4). Actualmente las indicaciones para este tipo de cirugía contemplan no solamente lo relacionado con la infertilidad, sino también la mayoría de la patología ginecológica abdominal, encontrándose la Endoscopia Ginecológica operatoria aprobada en los diferentes manuales de tarifas médicas $(3,5)$.

El tratamiento y el pronóstico de muchas patologías ginecológicas depende en gran parte de la extensión y severidad de las mismas, además del tipo de cirugía realizado y de los resultados obtenidos mediante estos procedimientos quirúrgicos (por ejemplo endometriosis o adherencias tubáricas en pacientes con infertilidad) (67); por consiguiente no sólo se deben unificar los parámetros de clasificación de la patología encontrada, sino que también es indispensable contar con un instrumento descriptivo adecuado que evite al máximo los errores de interpretación.

Aunque es posible desarrollar una videoteca de los procedimientos filmados (8), no es práctico anexar los videos a todas las historias clínicas o remitir solamente la película al médico tratante que solicitó la Laparoscopia. El editar la cinta de video, bien sea intraoperatoriamente o posteriormente, implica un consumo adicional de tiempo que difícilmente se puede mantener de manera prolongada. Por lo tanto un informe escrito completo y exacto de la Endoscopia Ginecológica sigue siendo indispensable para el adecuado manejo de las pacientes, máxime cuando se tiene en cuenta que muchos ginecólogos tratarán a las pacientes basados únicamente en el informe escrito.

Aunque no existe el informe ideal que reúna todas las características y soluciones todos los problemas, el modelo presentado puede resultar de mucha utilidad al mejorar la descripción al mismo tiempo que reduce el tiempo de elaboración. 
La sección anotada como Código se refiere a la codificación del diagnóstico preoperatorio, a los diagnósticos intraoperatorios o al código del procedimiento terapéutico realizado. Para designar este último se sugiere utilizar, en razón de su difusión en todo el territorio Colombiano, los códigos encontrados en el Manual de Procedimientos y Tarifas del Instituto de Seguro Social, vigente, colocando un código nuevo para los pocos procedimientos que no se encuentren en dicho Manual. Próximamente la Comisión Permanente en Endoscopia Ginecológica de la S.C.O.G. estará publicando el listado completo de los procedimientos realizados en la actualidad en este campo, junto con la codificación recomendada tanto para la parte diagnóstica como la terapéutica. Llenar correctamente estas casillas permitirá contar con un instrumento descriptivo y de análisis que podrá codificarse, guardarse y estudiarse rápidamente. Salta a la vista que codificar los informes de esta forma brindará la posibilidad de unificar la información de manera precisa, facilitando los estudios comparativos, bien sea interinstitucionales, entre diversas regiones del país etc.

Finalmente se sugiere utilizar en este informe laparohisteroscópico las clasificaciones ya conocidas de The American Fertility Society para endometriosis, adherencias anexiales y uterinas, lesiones tubáricas, maiformaciones mullerianas y dolor pélvico $(7,9-10)$, teniendo presentes las recomendaciones de Candiani para la clasificación adecuada de la endometriosis (11). A causa de los numerosos parámetros que se deben tener en cuenta de manera rutinaria, ya no es práctico intentar incluir en cada hoja de informe un resumen de las clasificaciones mencionadas. Se sugiere dejar en el sitio en que el cirujano debe llenar el lnforme de Endoscopia Ginecológica una carpeta que las incluya, disponible para consulta inmediata.

\section{Conclusión}

El modelo de Informe de Endoscopia Ginecológica presentado aquí y recomendado por la Comisión Permanente en Endoscopia Ginecológica de la Sociedad Colombiana de Obstetricia y Ginecología, tiene las siguientes ventajas:

1. Permite anotar una mayor cantidad de información gráfica y escrita, haciéndolo más exacto.

2. Disminuye el trabajo que representa su elaboración al concentrarse en los aspectos anormales encontrados.

3. Permite correlacionar los aspectos diagnósticos y operatorios del procedimiento.

4. Da una imagen clara de la totalidad del procedimiento, desde el inicio hasta el control postoperatorio y el resultado de Patología, constituyendo un conjunto integral muy útil en casos de remisión de resultados, de análisis o control por parte de otros médicos.

5. De difundirse su utilización a nivel nacional se constituiría en un paso indispensable hacia la unificación de la información en Endoscopia Ginecológica. Esto permitiría análisis comparativos no sólo entre diferentes instituciones, sino también entre diferentes regiones del país.

6. Se sugiere utilizar las clasificaciones descriptivas de The American Fertility Society para endometriosis, adherencias anexiales y uterinas, lesiones tubáricas, malformaciones müllerianas y dolor pélvico.

\section{BIBLIOGRAFIA}

1. Gaskin TA., Isobe JH., Mathews JL., Winchester SB., Smith RJ. Laparoscopy and the General Sugeon. Surg. Clin. North. Am. 1991; 71: 1085-1097.

2. Broussard TD., Mckernan JB., Laws HL. Horizons in Endoscopic Surgery. En: Braverman MH., Tawes RL., eds. Surgical Technology International II. 1a. ed. San Francisco, California. Editado por Surgical Technology International 1993; 37-39.

3. Instituto de Seguros Sociales. Manual de Tarifas. Acuerdo Número 113 de 1996.

4. Awwad JT., Isaacson K. Indications and Contraindications of Laparoscopy. En: Cohen SM., ed. Operative Laparoscopy and histeroscopy. 1a. ed. New York. Churchill Livingstone Inc. 1996; 519.

5. Federación Colombiana de Sociedades de Obstetricia y Ginecología. Manual de Codificación y Honorarios para Procedimientos Ginecológicos Obstétricos. Fecolsog 1996; 18-19.
6. Speroff L., Glass RH., Kase NG. Endometriosis. En: Clinical Gynecologic Endocrinology and infertility. Fifth Edition Baltimore. Williams \& Wilkins 1994; 853-71.

7. The American Fertility Society, Classifications of anexal adhesions, distal tubal occlusion, tubal occlusion secundary to tubal ligation, tubal pregnancies, müllerian anomalies and intrauterine adhesions, Fertil Steril 1989; 49: 944

8. Levinson CJ. Documentation for Endoscopy. En. Soderstrom RM., ed. Operative Laparoscopy. The Master's Techniques 1a. ed. New York. Raven Press, 1993; 203-205.

9. The American Fertility Society. Revised American Fertility Society classification of endometriosis. Fertil Steril 1985; 43: 351.

10. The American Fertility Society. Management of endometriosis in the presence of pelvic pain. Fertil Steril 1993; 60: 952.

11. Candiani GB., Vercellini P., Fedele L. Laparoscopic ovarian puncture for correct staging of endometriosis Fertil Steril 1990; 53: 994. 


\section{INFORME DE ENDOSCOPIA GINECOLOGICA}

FECHA:

CLINICA:

CIRUJANO:

\section{PACIENTE:}

\begin{tabular}{l|l} 
Edad: & G \\
\hline
\end{tabular}

\begin{tabular}{|l|l}
\hline G & P \\
\hline
\end{tabular}

\begin{tabular}{l|l}
$\mathbf{P}$ & $\mathbf{A}$ \\
\hline
\end{tabular}

\begin{tabular}{l|l} 
A & FUM:
\end{tabular}

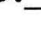

Antecedentes:

Diagnóstico Prequirúrgico:

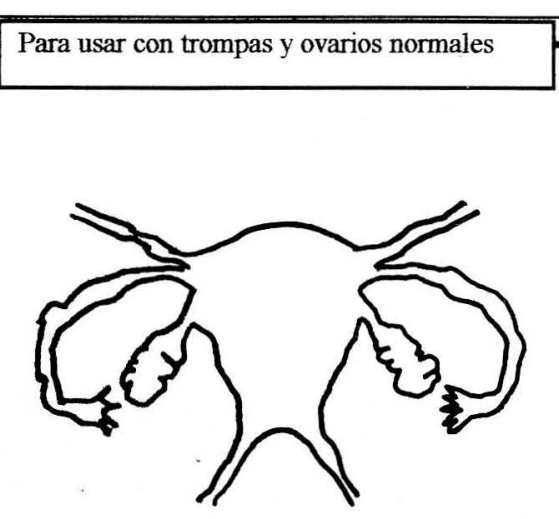

Para usar con trompas y ovarios anormales

Códigos:

\section{Cinta de Video: $\mathrm{Si}$}

No

H.C.:
Planif::

\begin{tabular}{l|l|l|}
\multicolumn{2}{l|}{} & H.C.: \\
\hline & Día Ciclo: & Planif.:
\end{tabular}

(1)




\begin{tabular}{|l|l|l|l|l|l|l|l|}
\hline 1.Douglas & & 2.Peritoneo Douglas & 3. Lig. Ut.Sacro Izdo & & 4.Lig. Ut.Sacro Der & 5. Otros & \\
\hline
\end{tabular}

\section{Otros Hallazgos:}

\section{DIAGNOSTICOS (Anotar códigos) Y PROCEDIMIENTOS REALIZADOS (Anotar códigos)}

\section{DESCRIPCION OPERATORIA:}

\begin{tabular}{|l|l|l|l|l|l|}
\hline \multicolumn{3}{|c|}{ HISTEROSCOPIA } \\
\hline
\end{tabular}

Controles Postoperatorios (Fecha:

): 